\title{
An Autostereoscopic Device for Mobile Applications Based on a Liquid Crystal Microlens Array and an OLED Display
}

\author{
José Francisco Algorri, Virginia Urruchi del Pozo, José Manuel Sánchez-Pena, \\ and
} José Manuel Otón

\begin{abstract}
In recent years, many experimental and theoretical research groups worldwide have actively worked on demonstrating the use of liquid crystals (LCs) as adaptive lenses for image generation, waveform shaping, and non-mechanical focusing applications. In particular, important achievements have concerned the development of alternative solutions for 3D vision. This work focuses on the design and evaluation of the electro-optic response of a $L C$-based $2 \mathrm{D} / 3 \mathrm{D}$ autostereoscopic display prototype. A strategy for achieving 2D/3D vision has been implemented with a cylindrical $L C$ lens array placed in front of a display; this array acts as a lenticular sheet with a tunable focal length by electrically controlling the birefringence. The performance of the $2 \mathrm{D} / 3 \mathrm{D}$ device was evaluated in terms of the angular luminance, image deflection, crosstalk, and 3D contrast within a simulated environment. These measurements were performed with characterization equipment for autostereoscopic 3D displays (angular resolution of $\left.\mathbf{0 . 0 3}^{\circ}\right)$.
\end{abstract}

Index Terms - Liquid crystal devices (LCDs), optical arrays, optical crosstalk, optical refraction, stereo vision.

\section{INTRODUCTION}

$\mathbf{M}$ ORE than 170 years ago, Charles Wheatstone demonstrated the first stereoscope to the Roy al Society (1838). He illustrated the basic principle of stereoscopic vision, the brain's capacity to achieve depth perception from different images. He combined mirrors to present separate images representing different perspectives of the same object for each eye. In the early 1900s, a new theory based on lenticular lenses applied this concept, but it was not until 1930 that the first "lenticular" images were produced by Victor Anderson. These images were of poor quality due to the production methods available at the time. More recently, this technology has improved considerably, primarily due to new manufacturing processes, advanced photolithography techniques, and the reduction of production costs. Furthermore, this technology can now display both three-dimensional and different styles of animated visual effects without the need for special viewing glasses. Interest has arisen in applying this technology, known as spatial multiplexing, to different areas, such as cinema, television, virtual prototyping, and medical imaging.

At present, most commercial 3D autostereoscopic devices use the spatial multiplexing technique. The operating principle is based on the optimization of two aspects: the correct capture of the necessary stereogram for display on the screen and the correct visualization of these images by the user. The stereograms are two images (corresponding to the same scene viewed from the two different angles of the right and left eyes) that are intertwined in columns of displayed pixels. At this point, a system capable of directing each column of pixels to the corresponding eye is necessary. Then, the brain interprets the signals and generates a stereoscopic response. There are two main approaches to achieving this function: parallax barriers [1] and lenticular systems [2]. A parallax barrier consists of masks that contain vertical apertures to cover the light path to the eye. The barrier can be considered a mature technology because commercial devices have been produced. For example, dynamic barriers based on liquid crystal displays (LCDs) are used to generate black and white columns. Despite its maturity, this technology has considerable drawbacks [3] such as lower brightness, small viewing angles, and crosstalk, which is caused by diffraction and is difficult to suppress. To improve the function of this system, lenticular technology can be used. In this work, we propose the use of a LC lenticular lens manufactured from novel materials to resolve the main drawback of lenticular technology, the tunability of the 3D distance.

The application of LC lenses has some advantages, such as weight reduction, a tunable focal distance, and low power. Until now, several topologies have been proposed. Notable solutions include patterned electrodes [4] and modal control [5]. The patterned electrode technique is based on voltage distribution through a dielectric layer. In one case, the electrodes are separated from the LC by a thin glass [6]. This method usually employs two high-voltage controls $(>50 \mathrm{~V})$. The other case surpasses these limitations and places the electrodes directly onto the LC surface. The optical properties depend on the relation between the lens aperture and the LC thickness [7] and require lenses smaller than 300-400 $\mu \mathrm{m}$. For larger diameters, the necessary thickness would be problematic. A technique that overcomes these aspects and simultaneously operates with only one low voltage is modal control. This technique 
consists of generating a radial graded voltage across each lens diameter with a high resistivity layer. For this technique, the layer is deposited onto the patterned electrode and is termed the control electrode. In this work, we propose a special mask for the electrode pattern (comb interdigitated) for a tunable cylindrical lenticular lens array with modal control. To date, different materials have been employed as control electrodes; for example, titanium oxide, PEDOT, and thin layers of ITO have been reported [8]. Problems arise due to the resistivity; these materials are limited to diameters larger than $1 \mathrm{~mm}$. The resistivity in these materials is usually approximately $1-10$ $\mathrm{M} \Omega / \mathrm{sq}$, which is too small to achieve a proper distribution of the fringe fields.

In this work, a cylindrical microlens array has been reported for the first time with a new manufacturing protocol based on a set of two layers (a dielectric layer of $\mathrm{SiO}_{2}$ and a metallic layer of nickel) for lenses with modal driving [9]. This array consists of a set of lenses, each $570 \mu \mathrm{m}$ in diameter whose behavior is based on a nanometric layer of nickel. This layer determines the voltage distribution across the lens diameter; without it, each lens would work as a hole-patterned lens and the voltage would drop at the lens edges. Modal lenses exhibit sometimes some spherical aberration because of the high resistivity of the manufactured layer. However, this aberration can be reduced by increasing the thickness of the layer [10]. We demonstrate how these lenses can work in an autostereoscopic system and validate, for the first time, the proposed system through 3D contrast measurements. Unlike other proposed systems [11]-[19], modal LC lenses have a wide range of focal length tunability, so this system could adjust the 3D distance over a wide range. Other improvement over other proposed LC lenses or the fixed lenticular systems is the capability of switching between 2D and $3 \mathrm{D}$ vision. It is also important to mention that other researchers are also working with modal control principle [20], [21]; in the referred works a system that enables rotation of the $3 \mathrm{D}$ image is demonstrated. Despite this, the resistivity is not high enough to create a voltage gradient when all electrodes are at the same voltage. They need a ground voltage at the center of each lens to have a voltage gradient, reducing the advantage of tunability of modal LC lenses.

\section{Design of AN Autostereoscopic Device}

The design of an autostereoscopic device involves several steps in which morphological (pupillary distance or view distance) and constructive (display parameters) factors must be considered. In this section, the design equations and the materials employed are described.

\section{A. Mask Design}

It is important to note that the size of the mask and the alignment with the pixel columns are the most critical points. Small differences in these parameters could cause high levels of crosstalk, which could render the lens array unusable for autostereoscopic vision. To manufacture LC lenses on a micrometric scale, a special mask is required for the photolithographic process. For this mask, an Autocad (Autodesk) design was etched in a chrome mask, which was $980 \AA$ thick, over a $5 \times 5$ inch square glass. The commercial OLED display

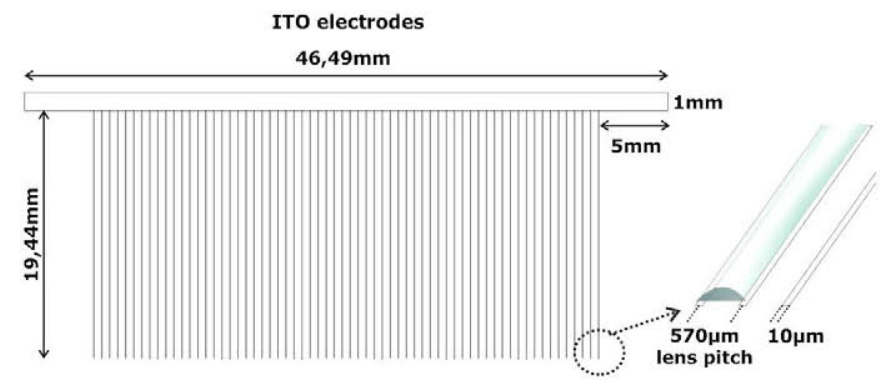

Fig. 1. Diagram of the electrode pattern etched on a chrome mask.

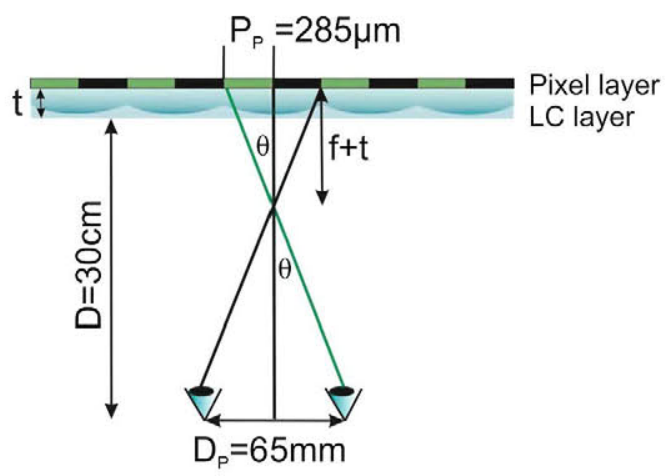

Fig. 2. Geometry for spatial multiplexing based on a lenticular lens array.

has the following characteristics: $1.6^{\prime \prime}$ in size, $128 \times 64$ pixel resolution and a pixel pitch size (pixel + interpixel) of $285 \mu \mathrm{m}$ $(255 \mu \mathrm{m}+35 \mu \mathrm{m})$. Considering that each lens is placed above two columns of pixels, the necessary diameter is $570 \mu \mathrm{m}$ per lens. For the proposed display, the designed mask is depicted in Fig. 1.

The patterned electrodes for a cylindrical lens array consist of parallel electrodes ( $10 \mu \mathrm{m}$ width) connected to the same potential. The main electrode has a connection of $1 \times 5 \mathrm{~mm}$. A total of 65 electrodes are used to create 64 microlenses. The photolithographic process is critical due to the sizes of the electrodes. In this case, all of the aspects of the process have been tested and optimized. The ground plane is simply a continuous plane of ITO.

\section{B. Viewing Distance}

In a mobile display, the distance required to properly view the $3 \mathrm{D}$ effect is not too large. The typical distance is usually between 20 and $35 \mathrm{~cm}$. The optimal distance for observing the autostereoscopic image determines the necessary focal distance (Fig. 2).

In Fig. 2, $D$ is the distance between the observer and the display, $P_{P}$ is the pixel pitch, $D_{P}$ is the interpupillary distance, and $\mathrm{t}$ is the thickness of the LC lens. By considering the extreme case in which the rays from the two endpoint pixels reach different eyes and for a $3 \mathrm{D}$ distance of $30 \mathrm{~cm}$, the focal distance can be extracted by trigonometry (Fig. 2)

$$
\left.\begin{array}{l}
\tan \theta=\frac{\frac{D p}{2}}{D-f} \\
\tan \theta=\frac{\frac{P p}{2}}{f+t}
\end{array}\right\} f=\frac{D \cdot P p-D p \cdot t}{D p+P p} \approx \frac{D \cdot P p}{D p}=1.3 \mathrm{~mm} .
$$




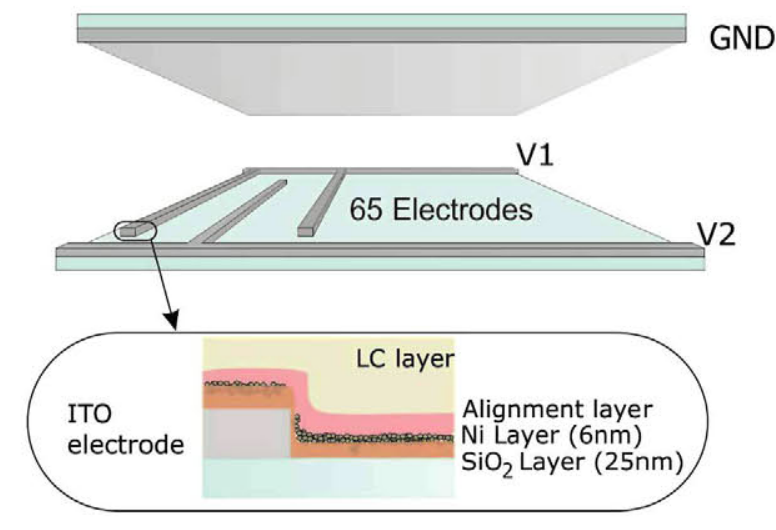

Fig. 3. 3D diagram with a cross-sectional detail of the lenticular array.

The last equation, which relates the 3D distance to the necessary focal distance for a microlens array placed in front of the display, is essential for the design of autostereoscopic devices based on microlenses. The use of a modal LC lens has many advantages over other technologies; one of the most important advantages is the control of aberrations via different harmonics in the applied voltage [22] or symmetric electrodes [23]. Another important characteristic is their focal length tunability in a broad range. For example, high birefringence materials can have a birefringence of 0.4 . For this diameter and a thickness of $100 \mu \mathrm{m}$, the minimum focal length would be $1 \mathrm{~mm}$. The focal length achieved with the maximum birefringence always increases with decreases in the applied voltage. In this case, the 3D distance, estimated by using (1), could be controlled from $22 \mathrm{~cm}$ to a few meters. Without voltage, the LC lens behaves as a transparent glass.

\section{Liquid Crystal Lenticular Lenses}

In this setup, the lens diameter determines the type of topology used. In this case, based on the pixel diameter of $285 \mu \mathrm{m}$, the lenses require a diameter of $570 \mu \mathrm{m}$, so the hole-patterning technique is not suitable and the materials previously reported in the modal technique are not appropriate. As mentioned previously, in this work, we propose a new type of material for a modal LC lens. A deposition layer of $\mathrm{SiO}_{2}$ $(250 \AA)$, and subsequently, a metallic layer of nickel ( $60 \AA)$ is placed by evaporation over the ITO electrode. Previous studies have clearly demonstrated nonlinear resistivity as a function of the material thickness at the nanometer scale [24]. Nickel was chosen because of its low cost and the ease with which it can be deposited through an evaporation process. Some recent works have also demonstrated how the inclusion of a $\mathrm{SiO} 2$ layer could improve the resistivity of the set by several orders of magnitude, produced by the metal-induced crystallization process [25]. We have experimentally observed how these two layers set increases the resistivity exponentially if the thickness of the evaporated nickel is smaller than $7 \mathrm{~nm}(1-5 \mathrm{G} \Omega / \mathrm{sq})$ [10]. In Fig. 3, a cross-sectional view of these layers is shown.

When a potential is applied to the ITO electrode, this layer forms a voltage gradient between the electrodes. This voltage gradient changes the molecular director across the lens diameter and produces a gradient in the refraction index. Because
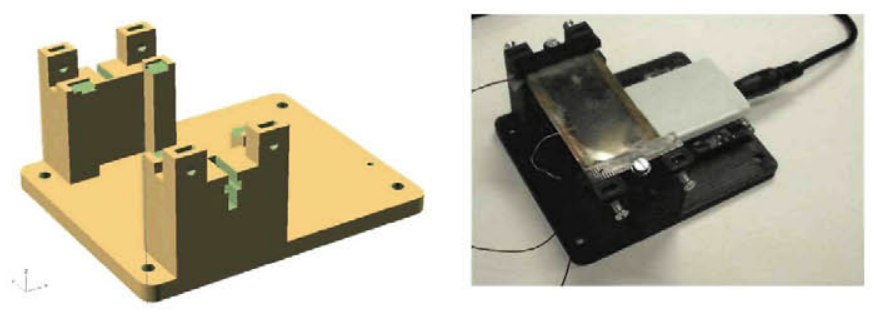

Fig. 4. Lenticular positioner. (a) OpenSCAD design and (b) final prototype.

LC lenses are actually GRIN (GRadient INdex) lenses, the estimation of the focal length, $f_{\mathrm{GRIN}}$, of the LC lenses is simple, if the focusing of parallel rays is considered

$$
f_{\mathrm{GRIN}}=\frac{r^{2}}{2 \cdot d \cdot \Delta n}
$$

The focal length $\left(f_{\text {GRIN }}\right)$ and diameter $(2 \mathrm{r})$ have been determined in previous sections, so only two adjustable variables, the thickness and birefringence, remain. The birefringence is a parameter determined by the material; the commercial nematic liquid crystal MDA-98-1602 from Merck was selected because of its high birefringence, $\Delta n=0.2666$. Therefore, only one adjustable parameter, the thickness, remains. Based on (2), the necessary thickness is $117 \mu \mathrm{m}$. In this case, two different lenticular devices, with thicknesses of $100 \mu \mathrm{m}$ and $50 \mu \mathrm{m}$, were manufactured. The LC lenticular array is placed on top of the 2D OLED display so that each column of pixels of the display is covered with each lens of the array as shown in Fig. 2.

\section{Prototype}

An important advantage of OLED displays is their linear polarization, which is parallel to the pixel columns. This polarization is used to cancel light reflection from internal electrodes. The LC arrays have the alignment layers in the electrode direction (antiparallel configuration), so the molecules are aligned with the polarization of light and take advantage of the LC birefringence. This advantage saves the inclusion of a single polarizer in the prototype. Once the $\mathrm{LC}$ lenses are set at the correct dimensions, according to the pixel size, the most important step is to place each lens over two columns of pixels. For this process, a lenticular positioner was designed with OpenSCAD and was realized with a 3D printer (Fig. 4). This device consists of a fixed base and two adjustable pieces that allow the horizontal and vertical positions to be precisely adjusted. The lenticular lens is controlled by an $\mathrm{AC}$ voltage $(1 \mathrm{kHz})$ to preserve the LC characteristics (to avoid the concentration of ions). A simple driver, based on a PIC $18 \mathrm{~F} 45 \mathrm{k} 20$ microcontroller and LM392 comparators, generates square waveforms with tunable frequencies and amplitudes. The entire system is portable if it is supplied with batteries.

\section{ElECTRO-OPTIC ChARACTERIZATION}

Electro-optic characterization related to $3 \mathrm{D}$ vision requires special equipment. This equipment must have the ability to measure light at different angles. To date, only simple characterizations of autostereoscopic systems have been described in 


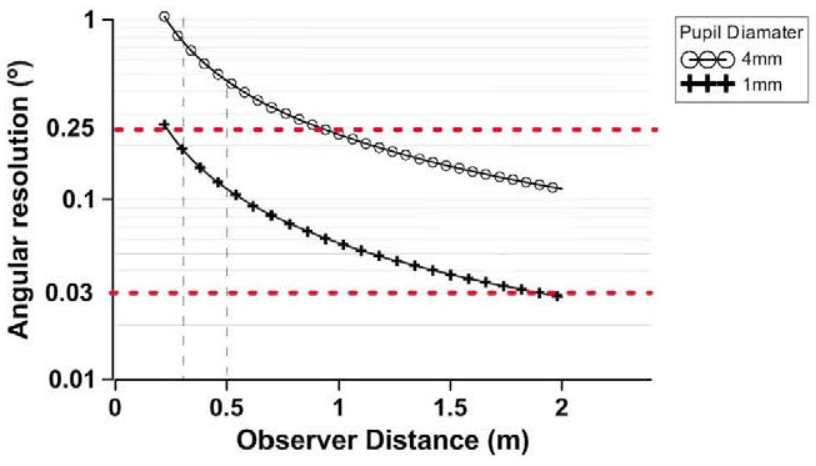

Fig. 5. Angular resolution as a function of $3 \mathrm{D}$ distance.

the literature [26]. These characterizations are based on photodetectors that are movable along one axis at the optimum 3D distance. Components such as goniometers (movable photometers), which are typically used for displays, have been used; however, such systems have limited angular resolution and position. It may be assumed that these limitations will render the measurements inaccurate in multiview autostereoscopic systems. The main problem in evaluating autostereoscopic displays is discerning which image reaches a particular eye. For this determination, it is very important to distinguish between two rays of incoming light (from a point on the 3D display) with similar directions. Moreover, the uncertainty of the position in the measurement must be smaller than the pupil size (1-4 mm) to make this measurement acceptable, otherwise, the exact information for one eye cannot be known. As shown in Fig. 2, the corresponding angle of the pupil is $\operatorname{atan}\left(t_{p} / D\right)$, where $t_{p}$ is the pupil size. Fig. 5 presents the relation between this angle, which is equal to the minimum angular resolution, and the observer distance $(D)$.

In a mobile application, an observer distance of $0.3-0.5 \mathrm{~m}$ can be considered (vertical dotted line). If the more restrictive condition is used (pupil size of $1 \mathrm{~mm}$ ), the minimum angular resolution is $0.19^{\circ}$ to $0.11^{\circ}$. For such small resolutions, goniometers cannot be employed, and typical resolutions range from $0.25^{\circ}$ (PM-FPMS) to $1^{\circ}$ (GC 5000L). In contrast, measurement devices based on Fourier optics usually have a minimum resolution of $0.25^{\circ}$ (Ezcontrast XL88MS). This situation requires the use of equipment with resolutions of less than $0.11^{\circ}$. The only equipment on the market that fits these characteristics is the VCMaster3D, which is specifically designed and manufactured by ELDIM for autostereoscopic applications. For an angular resolution of $0.03^{\circ}$, the observer distance can be as high as $2 \mathrm{~m}$ for pupil sizes of $1 \mathrm{~mm}$ and $7.6 \mathrm{~m}$ for pupil sizes of $4 \mathrm{~mm}$.

\section{A. Angular Luminance}

Based on the specifications, the autostereoscopic device is placed $1.5 \mathrm{~cm}$ in front of the equipment. With this equipment, the angular luminance from the lenticular $\mathrm{LC}$ lenses is measured with an angular aperture of $\pm 50^{\circ}$ (Fig. 6).

Light is uniformly distributed in the OLED display. To evaluate the quality of light deviation at different angles, a single special image has been employed. This image is composed of two interlaced images; one column of pixels is black, and the adjacent column is green. The objective is to illuminate only

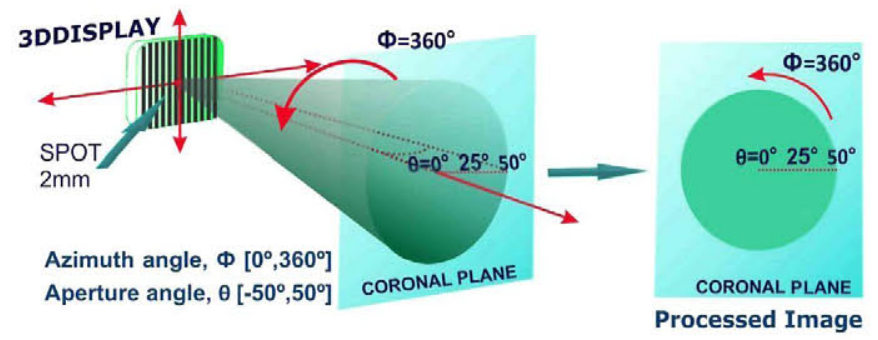

Fig. 6. Capture mode of the VCMaster3D.

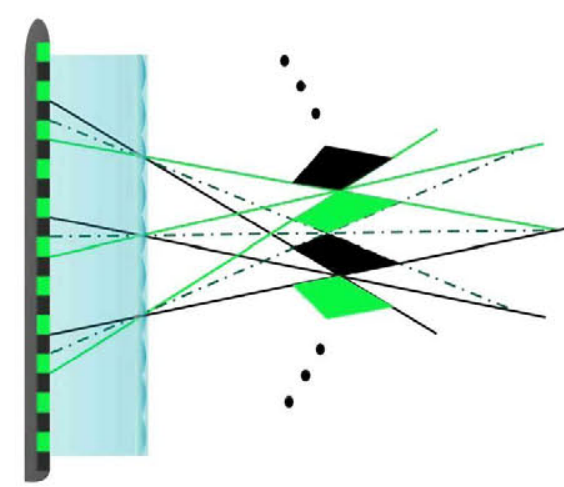

Fig. 7. Diagram of the viewing zones caused by a black-white pattern

half of each lens and to determine the light deviation. In this approach, the two different views are a black image and a green image for the right and left eyes, respectively. The expected result is depicted in Fig. 7.

The first experiment performed is an angular luminance measurement for different voltages applied to the LC lenticular sheet. In Fig. 8, the light can be observed to deviate at different angles. Different intensity patterns are obtained as a function of the voltage.

If no voltage is applied, the light does not deviate. All of the molecules in the LC lenticular array are in the same position, so the array behaves like a common glass. When the threshold voltage is exceeded, a progressive variation in the tilt angle of the $\mathrm{LC}$ molecules appears. A response time of $0.5 \mathrm{~s}$ has been measured in this case (this time is determined by the thickness of the LC layer). This variation produces a gradient in the refractive index and concentrates the light at the focal point. These lenses still have high spherical aberration, so the concentration of light is not high. At $7.5 \mathrm{~V}$, we have the optimum lens; in this case, the concentration of light is high (yellow areas), and there are clear separations between the views (blue areas). When this voltage is exceeded, the saturation of molecules at the ends of the lens causes a shrinking effect, allowing light to shine through and causing a decrease in the concentration of light. Additionally, the directivity of the light at different angles is loose.

\section{B. Crosstalk}

In autostereoscopic vision, the crosstalk parameter is used to measure the quality of the generated stereoscopic image. This parameter was first introduced by Montgomery in 2001 [27]. Each eye must see a different image. Nevertheless, when the right eye (or left eye) receives information intended for the left 


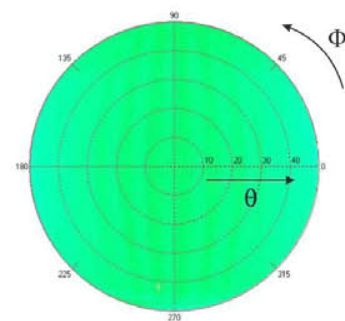

a)

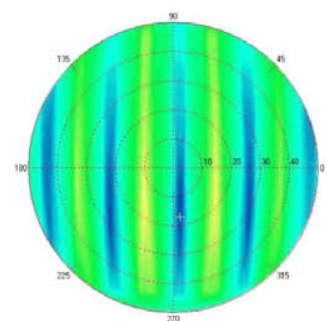

c)

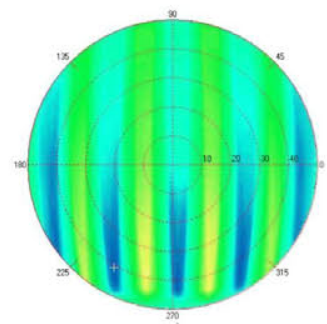

e)

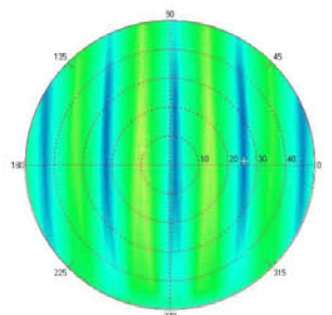

b)

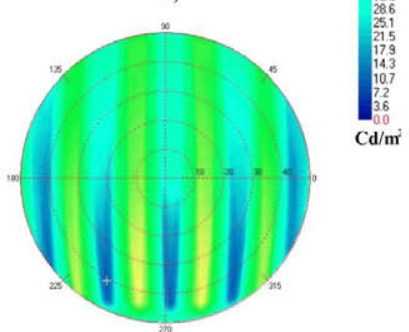

d)

f)

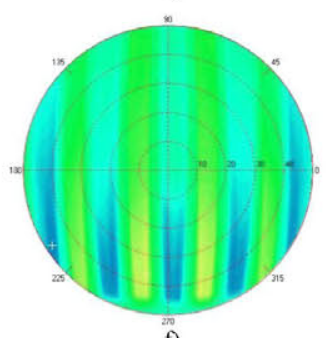

Fig. 8. Viewing zones $\left(\mathrm{cd} / \mathrm{m}^{2}\right)$ caused by a black-white pattern for different voltages applied to the LC lenticular sheet. (a) $0 \mathrm{~V}$, (b) $5 \mathrm{~V}$, (c) $7.5 \mathrm{~V}$, (d) $10 \mathrm{~V}$, (e) $15 \mathrm{~V}$, and (f) $20 \mathrm{~V}$.

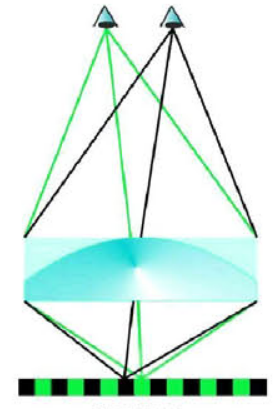

IDEAL

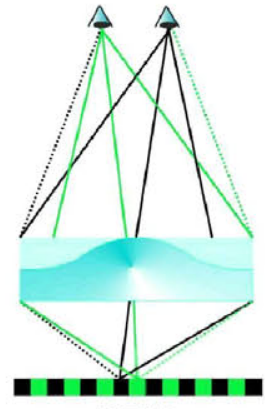

REAL
Fig. 9. Diagram for the crosstalk produced in ideal and real cases.

eye (or right eye), crosstalk $(\chi)$ is considered to occur. This parameter is defined as the relation between two luminances, corresponding to the incorrect image and the correct image (Fig. 9). In an ideal case and with correct implementation, that is, at the correct focal distance for the specifications, there is a distance (1) at which an observer will see white columns with one eye and black columns with the other eye. In this case, no unintended light reaches the eye. In contrast, if the lens has some type of defect, some light reaches the wrong eye.

To quantify this parameter, a second experiment is performed. In this case, two images (with two interlaced images each) are used. The first case is the same as in the previous experiment. The second case is the opposite of the first in order to place the maximum intensity in the other view. The luminance of each

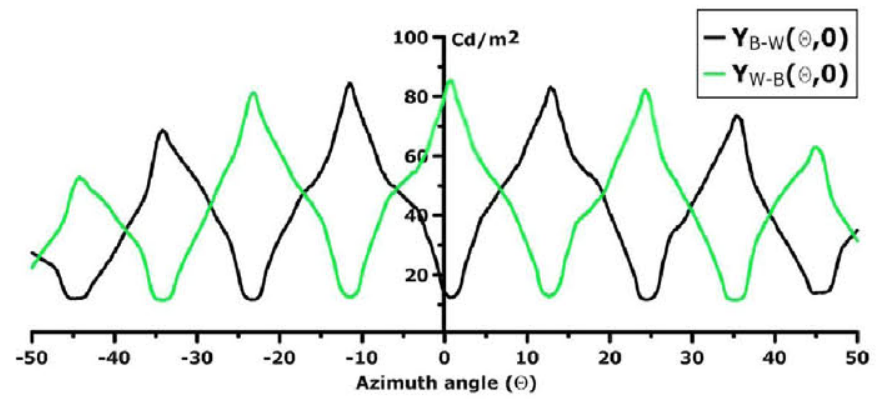

Fig. 10. Diagram for the crosstalk produced in the ideal and real cases.

one is defined as $\mathrm{Y}_{\mathrm{B}-\mathrm{W}}$ and $\mathrm{Y}_{\mathrm{W}-\mathrm{B}}$. The voltage applied to the lenticular sheet in Fig. 10 is $7.5 \mathrm{~V}_{\text {rms }}$.

In Fig. 10, the angular luminance at the azimuth angle of $0^{\circ}$ is represented for the two different images $\left(\mathrm{Y}_{\mathrm{B}-\mathrm{W}}\right.$ and $\left.\mathrm{Y}_{\mathrm{W}-\mathrm{B}}\right)$. In this figure, we can observe the deviation in each half lens of the lenticular sheet relative to the other half. Additionally, the crosstalk can be obtained as a function of the viewing angle. For example, for an angle of $2^{\circ}$, only the light of the white-black image should be observed. However, there is a small luminance from the black-white image at the same position that interferes with the previous image. In this case, the first pattern (whiteblack) has almost $90 \mathrm{~cd} / \mathrm{m}^{2}$, and the other pattern (black-white) is approximately $10 \mathrm{~cd} / \mathrm{m}^{2}$, so the experimental crosstalk is approximately (3) $10 \%$.

The main cause of the measured crosstalk is light falling on the CCD from wrong pixels. This light deviation could be minimized by adjusting the resistivity of the layer in order to exploit the quadratic range of the LC birefringence. To this end, the deposition of the Nickel in the $\mathrm{SiO}_{2}$ layer must be further investigated. A slight decrease in the resistivity is suggested for the reduction of the spherical aberration produced by a flatness effect in the lens center. Another cause of crosstalk is the deviated position of each lens of the array from the corresponding two columns of the 2D display. It can be corrected using a positioner system with micrometric movement resolution. Despite this, the crosstalk is small enough to clearly see two different images in the two eyes (Fig. 13) and to experience the autostereoscopic effect.

\section{Angular Crosstalk}

For this experiment, four images with different patterns are used. One image is white-black and another is black-white to measure the right and left views. Additionally, black-black and white-white are used to determine the on-off transmission. Although the VCMaster3D is placed at $1.5 \mathrm{~cm}$, these four measurements of the angular luminance allow the contrast associated with each eye to be estimated and allow the distribution of light to be calculated at a particular position for the left view, right view, and 3D contrast (the distribution of the 3D autostereoscopic effect). The angular contrast for each view is [28]

$$
\begin{gathered}
C_{R}(\theta, \varphi)=\frac{Y_{\mathrm{BW}}(\theta, \varphi)-Y_{\mathrm{BB}}(\theta, \varphi)}{Y_{\mathrm{WB}}(\theta, \varphi)-Y_{\mathrm{BB}}(\theta, \varphi)}=\frac{1}{\chi_{R}} \\
C_{L}(\theta, \varphi)=\frac{Y_{\mathrm{WB}}(\theta, \varphi)-Y_{\mathrm{BB}}(\theta, \varphi)}{Y_{\mathrm{BW}}(\theta, \varphi)-Y_{\mathrm{BB}}(\theta, \varphi)}=\frac{1}{\chi_{L}}
\end{gathered}
$$




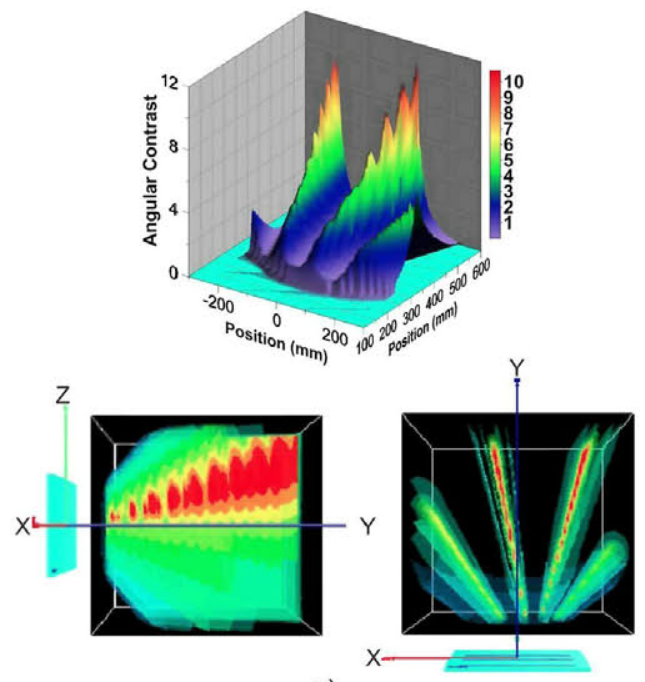

a)

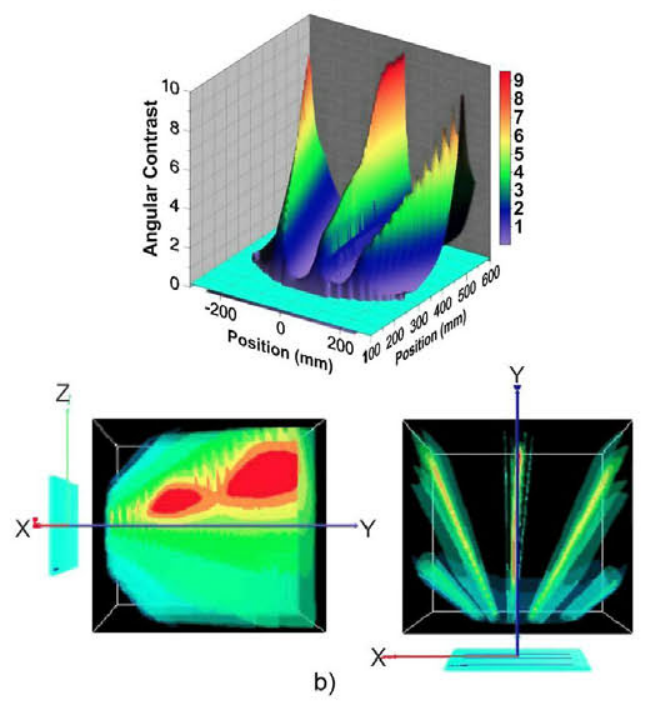

b)

Fig. 11. Angular contrast for (a) right and (b) left views.

where $Y_{\mathrm{BW}}$ is the luminance of the black-white image in the polar coordinates determined by $\theta, \varphi$, and so on. The employed equations are the inverse of the crosstalk equation; however, this measure is standard in display applications. The 3D quality is optimized when the $C_{R}$ and $C_{L}$ are maximized at the same point; therefore, the $3 \mathrm{D}$ contrast can be estimated by

$$
C_{3 \mathrm{D}}(\theta, \varphi)=\sqrt{C_{R}\left(\theta_{R}, \varphi_{R}\right) \cdot C_{L}\left(\theta_{L}, \varphi_{L}\right)} .
$$

The product is used because good contrast is simultaneously required for each eye to obtain reasonable quality [29]. The square root maintains the dimensionality of the quantity for comparison with other display standards. Following these equations, the spaces for the angular contrast distributions are displayed in Fig. 11.

In the first diagram, a 3D graphic presents the angular contrast $\left(C_{R}, C_{L}\right)$ in the $\mathrm{XY}$ plane. Smoothing has been applied to improve the comprehensibility of the figure. In the other figures, the autostereoscopic display is represented by a small blue rectangle, and the angular contrast is represented in the $x, y$, and $z$ coordinates in a simulated environment. The color scale ranges from blue to red for low and high contrast, respectively.

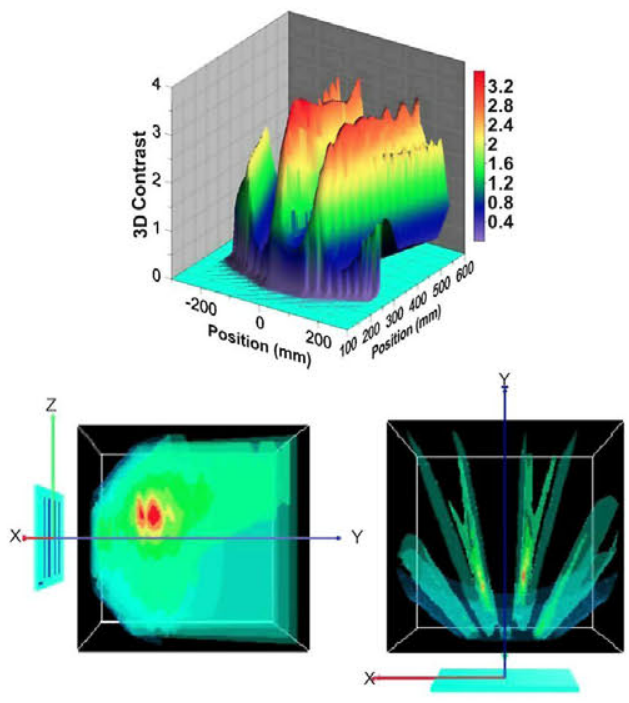

Fig. 12. 3D angular contrast.

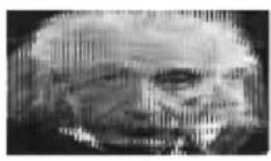

a)

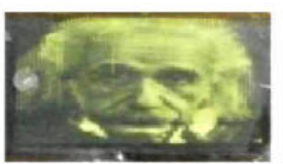

b)

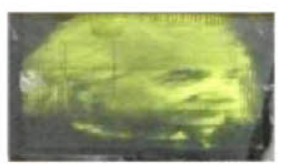

Fig. 13. (a) Interlaced image. (b) Two captures at different angles.

This measure indicates the angles and positions at which the left view and the right view can be observed with better quality. In this case, the left view can be observed at three different angles. The best position is located in front of the display, slightly inclined from the $\mathrm{XY}$ plane. The right view can be observed at $15^{\circ}$ relative to the $\mathrm{YZ}$ plane and at the same inclination from the $\mathrm{XY}$ plane as for the left view. From the previous data and (3), the $3 \mathrm{D}$ contrast $\left(C_{3 \mathrm{D}}\right)$ is obtained.

In Fig. 12, the red areas also indicate the positions at which the autostereoscopic effect has the best quality. For the same voltage at the $\mathrm{LC}$ lenticular sheet $\left(7.5 \mathrm{~V}_{\mathrm{rms}}\right)$, there are two clear viewing zones at $-15^{\circ}$ and $15^{\circ}$ relative to the $\mathrm{YZ}$ plane and at a distance between 30 and $35 \mathrm{~cm}$. This distance agrees well with the previous design. There are two other viewing zones at $-45^{\circ}$ and $45^{\circ}$; however, the 3D contrast is very low. This contrast ratio, which is inversely proportional to the crosstalk, can be improved in the manner described in the previous section.

\section{Image Capture}

To illustrate the previous measurements, different captures were acquired with a digital camera. As shown in Fig. 11, each view deviates at different angles. To measure this luminance, only columns of pixels switched on and off were required. In this final experiment, two images interlaced in one file were displayed in the autostereoscopic display. These images are not designed to create an autostereoscopic effect, but are sufficiently different to be clearly differentiable.

As shown in Fig. 13, two different images are observed when the viewing angle is changed. If autostereoscopic images are required, this content must be generated with a special camera. The major requirement for this camera is the inclusion of two different objectives in the same transverse plane (like two eyes). 
In this case, the content is generated with a commercial 3D camera by processing the MPO files. Then, with specialized software, the two different images corresponding to each eye are interlaced. The obtained results are preliminary but demonstrate how a lenticular LC lens array could be applied in the next generation of autostereoscopic displays.

\section{CONCLUSION}

A cylindrical microlens array has been reported for the first time with a new manufacturing protocol based on a set of two layers (a dielectric layer of $\mathrm{SiO}_{2}$ and a metallic layer of nickel) for lenses with modal driving. A 2D/3D switchable prototype based on an OLED display, LC microlenses, and a lenticular positioner has been designed and manufactured. The luminance measurements show $10 \%$ crosstalk between the views. The position between the display and the LC lenses is critical to the reduction of this parameter. The angular contrast measurements reveal an optimum distance of $30 \mathrm{~cm}$ to $35 \mathrm{~cm}$ for 3D vision. By optimizing the structural parameters and improving the lenticular positioner, a tunable autostereoscopic display with low crosstalk could be manufactured.

\section{REFERENCES}

[1] K. Kusunoki, M. Kaneyasu, H. Harada, H. Katagiri, K. Moriya, H. Ohnuma, Y. Hirakata, J. Koyama, S. Yamazaki, Y. Shima, and D. Kurosaki, "3D Display using active liquid crystal parallax barrier with supersonic position detector," in SID Int. Symp. Dig. Tech., Jun. 2012, vol. 43, no. 1, pp. 217-220.

[2] R. B. Johnson and G. A Jacobsen, "Advances in lenticular lens arrays for visual display," Proc. SPIE, vol. 5874, pp. 1-11, Aug. 2005.

[3] J. Mather, "3D TV without glasses," Phys. World, vol. 24, no. 6, pp. 33-36, Jun. 2011.

[4] Y.-H. Lin, H. Ren, K.-H. Fan-Chiang, W.-K. Choi, S. Gauza, X. Zhu, and S.-T. Wu, "Tunable-focus cylindrical liquid crystal lenses," Jpn.J. Appl. Phys., vol. 44, no. 1A, pp. 243-244, Jan. 2005.

[5] A. F. Naumov, G. D. Love, M. Y. Loktev, and F. L. Vladimirov, "Control optimization of spherical modal liquid crystal lenses," Opt. Express, vol. 4, no. 9, pp. 344-352, Apr. 1999.

[6] M. Ye, B. Wang, and S. Sato, "Realization of liquid crystal lens of large aperture and low driving voltages using thin layer of weakly conductive material," Opt. Express, vol. 16, no. 6, pp. 4302-4308, Mar. 2008.

[7] S. Sato, "Applications of liquid crystals to variable-focusing lenses," Opt. Rev., vol. 6, no. 6, pp. 471-485, Nov. 1999.

[8] N. Fraval and J. L. de Bougrenet de la Tocnaye, "Low aberrations symmetrical adaptive modal liquid crystal lens with short focal lengths," Appl. Opt., vol. 49, no. 15, pp. 2778-2783, May 2010.

[9] V. Urruchi, J. F. Algorri, M. A. Geday, X. Quintana, N. Bennis, and J. M. Sánchez-Pena, "Lenticular arrays based on liquid crystals," presented at the CLC'11, Międzyzdroje, Poland, Feb. 2011, unpublished.

[10] V. Urruchi, J. F. Algorri, J. M. Sánchez-Pena, N. Bennis, M. A. Geday, and J. M. Otón, "Electrooptic characterization of tunable cylindrical liquid crystal lenses," Mol. Cryst. Liq. Cryst., vol. 553, no. 1, pp. 211-219, Jan. 2012.

[11] Y. P. Huang, L. Y. Liao, and C. W. Chen, "2-D/3-D switchable autostereoscopic display with multi-electrically driven liquid-crystal (MeD-LC) lenses," J. Soc. Inf. Display, vol. 18, no. 9, pp. 642-646, Jun. 2010.

[12] H. K. Hong, S. M. Jung, B. J. Lee, and H. H. Shin, "Electric-field-driven LC lens for 3-D/2-D autostereoscopic display,"J. Soc. Inf. Display, vol. 17 , no. 5 , pp. $399-406$, Sep. 2009.

[13] Y. P. Huang and C. W. Chen, "Superzone fresnel liquid crystal lens for temporal scanning auto-stereoscopic display," J. Display Technol, vol. 8, no. 11, pp. 650-655, Nov. 2012.

[14] C. W. Chen, Y. P. Huang, and P. C. Chen, "Dual direction overdriving method for fast switching liquid crystal lens on $2 \mathrm{D} / 3 \mathrm{D}$ switchable autostereoscopic display," J. Display Technol., vol. 8, no. 10, pp. 559-561, Oct. 2012.
[15] H. K. Hong, S. M. Jung, B. J. Lee, and H. H. Shin, "Electric-field-driven LC lens for 3D/2D autostereoscopic display," J. Soc. Inf. Display, vol. 17, no. 5, pp. 399-406, May 2009.

[16] J. G. Lu, X. F. Sun, Y. Song, and H. P. D. Shieh, "2-D/3-D switchable display by Fresnel-type LC lens," J. Display Technol., vol. 7, no. 4, pp. 215-219, Apr. 2011.

[17] M. P. C. M. Krijn, S. T. de Zwart, D. K. G. de Boer, O. H. Willemsen, and M. Sluijter, "2-D/3-D displays based on switchable lenticulars," $J$. Soc. Inf. Display, vol. 16, no. 8, pp. 847-855, Jun. 2008.

[18] B. J. Mun, J. H. Baek, J. H. Lee, B. K. Kim, H. C. Choi, J. H. Kim, and G. D. Lee, "Low cell gap polymeric liquid crystal lens for $2-D / 3-D$ switchable auto-stereoscopic display," IEEE Trans. Electron. Devices, vol. 60 , no. 10 , pp. $3430-3434$, Oct. 2013.

[19] J. Harrold and G. J. Woodgate, "Autostereoscopic display technology for mobile 3DTV applications," Proc. SPIE, vol. 6490, 2007.

[20] Y. P. Huang et al., "Adaptive liquid crystal lens (LC-Lens) array for 3D display and capturing," presented at the DM2C'12, Miami, FL, USA, 2012.

[21] Y. Chang, T. Jen, C. Ting, and Y. Huang, "High-resistance liquidcrystal lens array for rotatable 2D/3D autostereoscopic display," Opt. Express, vol. 22, pp. 2714-2724, Feb. 2014.

[22] A. F. Naumov, G. D. Love, M. Y. Loktev, and F. L. Vladimirov, "Control optimization of spherical modal liquid crystal lenses," Opt. Express, vol. 4, no. 9, pp. 344-352, Apr. 1999.

[23] N. Fraval and J. L. de la Tocnaye, "Low aberrations symmetrical adaptive modal liquid crystal lens with short focal lengths," Appl. Opt., vol. 49 , no. 15 , pp. $2778-2783$, May 2010.

[24] A. I. Oliva Arias, "Nuevas propiedades físicas de materiales nanoestructurados," Ing. Rev. Acad., vol. 9, no. 3, pp. 39-46, Sep. 2005.

[25] M. A. Mohiddon, K. Lakshun Naidu, M. Ghanashyam Krishna, G. Dalba, and F. Rocea, "Growth, optical, electrical properties of silicon films produced by the metal-induced crystallization process," $J$. Nanopart. Res., vol. 13, no. 11, pp. 5999-6004, 2011.

[26] Y.-Y. Kao, Y.-P. Huang, K.-X. Yang, P. C.-P. Chao, C.-C. Tsai, and C. Mo, "An auto-stereoscopic 3D display using tunable liquid crystal lens array that mimics effects of GRIN lenticular lens array," in SID Int. Symp. Dig. Tech., Jun. 2009, vol. 40, no. 1, pp. 111-114.

[27] D. Montgomery, G. Woodgate, and A. Jacobs, "Analysis of the performance of a flat panel display system convertible between 2D and autostereoscopic 3D modes," Proc. SPIE, vol. 4297, Jan. 2001.

[28] P. Boher, T. Leroux, and T. Bignon, "A new way to characterize autostereoscopic 3D displays using Fourier optics instrument," Proc. SPIE, vol. 7237, Feb. 2009 .

[29] P. Boher, "Characterization of autostereoscopic 3D displays using Fourier optics viewing angle instrument," presented at the IDW '08, Niigata, Japan, Dec. 3-5, 2008.

José Francisco Algorri received the M.Sc. degree in electronic engineering from Universidad de Valladolid (Spain) in 2008, the M.Res. degree in advanced electronics systems from Universidad Carlos III de Madrid (Spain) in 2012, and is currently pursuing the Ph.D. degree in electronic engineering at Carlos III University of Madrid.

Since 2010 , he has been involved in research and development activities related to optical phase modulators based on Liquid Crystals at Universidad Carlos III de Madrid. He is also interested in interaction of nanoparticles and carbon nanotubes with liquid crystals.

Virginia Urruchi del Pozo received the M.Sc. and Ph.D. degrees in telecommunication engineering from the Universidad Politécnica de Madrid, Spain, in 1999 and 2004, respectively.

She has been an assistant professor at the Universidad Carlos III of Madrid from 2001 to 2009. Since 2010, she has been an associate professor. She has been involved in liquid crystals since 1998 and her research interests are the identification of new liquid crystal mixtures and technologies specifically for photonic (display and non-display) applications, electro-optic characterization, driving waveform design, and electrical simulation. She has contributed to the development of a national patent (Spain) about driving and method of fabrication of antiferroelectric liquid crystals displays without holding voltage. 
José Manuel Sánchez-Pena (M'95) was born in Lugo, Spain, in 1962. He received the M.S. and Ph.D. degrees in telecommunication engineering from the Polytechnic University of Madrid, Madrid, Spain, in 1988 and 1993, respectively.

From February to September 1993, he was a Visiting Researcher at Exeter University, U.K., engaged in the modeling of the electrooptical properties of liquid crystal displays (LCDs). In March 1995, he joined Carlos III University, Madrid, where he is currently an Associate Professor and Head of the Displays and Photonic Applications Group in the Electronics Technology Department. His main research interests include electrooptical characterization of liquid crystal displays, design of optical sensors, and optoelectronic instrumentation for rehabilitation technology applications and optical communications.

Dr. Pena is a member of the Society of Photo-Optical Instrumentation Engineers (SPIE), the International Liquid Crystal Society (ILCS), and the International Society for Augmentative and Alternative Communication (ISAAC).
José Manuel Otón received the Ph.D. degree in chemistry from the Universidad Complutense, Madrid, Spain, in 1976.

He is a Full Professor in the Department of Photonic Technology, School de Telecom Engineers, Universidad Politecnica de Madrid, Madrid, Spain, where he teaches courses on lasers, fiber optics, and optical communications. His current research interests include displays and photonic devices based on liquid crystals and on organic light-emitting devices (LEDs). He has coauthored over 100 scientific papers and presented over 150 communications to scientific conferences 